\title{
Outdoor swimming pools and the risks of asthma and allergies during adolescence
}

\author{
A. Bernard, M. Nickmilder and C. Voisin
}

\begin{abstract}
Exposure to indoor chlorinated swimming pools can be detrimental to the airways of swimmers and increase asthma risks but it is unknown whether these effects concern outdoor pools.
\end{abstract}

The present study examined 847 secondary school adolescents who had attended residential or nonresidential outdoor chlorinated pools at a variable rate. The main outcomes were: ever asthma (physician-diagnosed at any time); current asthma (ever asthma under medication and/or with exercise-induced bronchoconstriction); elevated exhaled nitric oxide; and aeroallergen-specific immunoglobulin (Ig)E in serum.

The prevalence of ever and current asthma significantly increased with the lifetime number of hours spent in outdoor pools by up to four and eight times, respectively, among adolescents with the highest attendance $(>500 \mathrm{~h})$ and a low exposure to indoor pools ( $<250 \mathrm{~h})$. Odds for asthma were significantly increased among adolescents with total serum $\operatorname{lgE}>25 \mathrm{kIU} \cdot \mathrm{L}^{-1}$, on average by 1-2 units for each 100-h increase in pool attendance. Use of residential outdoor pools was also associated with higher risks of elevated exhaled nitric oxide and sensitisation to cat or house dust mite allergens.

Outdoor chlorinated swimming pool attendance is associated with higher risks of asthma, airways inflammation and some respiratory allergies.

KEYWORDS: Aeroallergens, atopy, childhood asthma, exercise-induced asthma, exhaled nitric oxide, total immunoglobulin E

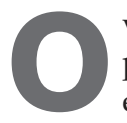
ver the last decades, outdoor swimming pools have become increasingly popular, especially in countries with a warm climate [1]. Global warming will probably see many more people installing private pools for exercising or refreshing on hot days. Most swimming pools worldwide are disinfected with chlorine-based disinfectants that in water release hypochlorous acid, a powerful oxidant that destroys pathogenic micro-organisms [2]. The type and form of chlorine used in swimming pools varies with the size of the pool and its level of attendance. Residential outdoor pools are usually sanitised with chlorinated isocyanurates, which are stabilised forms of chlorine that are easy to handle and resistant to ultraviolet degradation. Public outdoor pools use cheaper forms of chlorine, such as chlorine gas or sodium hypochlorite. However, chlorine as a swimming pool disinfectant presents two major drawbacks. First, upon oxidising organic substances originally from swimmers or other sources, hypochlorous acid generates a mixture of harmful breakdown products, which includes potent irritants, such as chloramines, haloacetic acids or haloacetonitriles [2, 3]. Secondly, another frequent drawback is that hypochlorous acid is a nonselective biocide that inevitably also reacts with the organs of the bather in contact with pool water or aerosols, causing irritation of the skin, eyes and upper respiratory tract [4-6].

Paradoxically, while the acute toxicity of chlorination products has been known for more than one century and populations of industrialised countries have been increasingly exposed to these chemicals, in particular with the development of swimming pools, studies evaluating their effects on swimmers have only recently started. Studies on elite swimmers were among the first to suggest that the chlorine-laden atmosphere of indoor pools could be detrimental to the lungs, by increasing the risk of asthma, bronchial hyperreactivy and airways inflammation [3, 7, 8]. Previous studies of children by the current authors attending indoor chlorinated swimming pools have shown that trichloramine, together with presumably aerosolised hypochlorous acid and chloramines, can damage the lung epithelium and promote the development of asthma, particularly
AFFILIATIONS

Dept of Public Health, Catholic University of Louvain, Brussels, Belgium.

CORRESPONDENCE

A. Bernard

Unit of Toxicology

Faculty of Medicine Catholic University of Louvain

Avenue E. Mounier 53.02

B-1200 Brussels

Belgium

Fax: 3227645328

E-mail: bernard@toxi.ucl.ac.be

Received:

September 022007

Accepted after revision:

May 072008

\section{SUPPORT STATEMENT}

A. Bernard is Research Director of the National Fund for Scientific Research (Brussels, Belgium). This work was supported by the National Fund for Scientific Research in Belgium, the Agency for Environmental and Occupational Health Safety in France and the Governments of the Walloon Region and of the French Community of Belgium (Belgium).

STATEMENT OF INTEREST

None declared. 
among children with higher concentrations of total serum immunoglobulin (Ig)E [9-12]. These effects might be responsible for the strong ecological associations that were recently reported between childhood asthma prevalence and the availability of indoor chlorinated swimming pools in Europe [13]. Other researchers have confirmed these adverse effects of pool chlorine on the airways of recreational swimmers, while providing further evidence that exposure to indoor chlorinated pools might contribute to the development of allergic diseases [14-16]. The chlorine compounds responsible for these respiratory effects are largely unknown. Currently, the most commonly suspected culprit is trichloramine, also called nitrogen trichloride, the gas that builds up in the air of indoor pools, giving them their distinctive chlorine smell. Trichloramine has been identified as a cause of asthma and respiratory problems in pool workers $[17,18]$. Swimmers, however, are mainly exposed when they actively inhale the volatile and aerosolised chlorination products in the air just above pool surface. Another potential source of exposure for swimmers, especially the youngest who are learning to swim, is the direct contact of the airways with chlorinated water that enters the upper respiratory tract and can be carried more or less deeply into the lungs, depending on the ventilation rate [12].

These uncertainties, regarding the chlorine compounds responsible for the respiratory problems in swimmers attending indoor pools, necessarily raise the question of the safety of open-air swimming pools. This question is especially important for countries with a warm climate, where backyard pools are very common and can be attended by children and their family almost all year round. The present epidemiological study focused on secondary school adolescents. The relationships between the attendance of outdoor pools, at home or during holidays, and the prevalence of asthma, respiratory allergies and airways inflammation have been explored using, whenever possible, objective outcome measures.

\section{MATERIALS AND METHODS}

\section{Study population}

Adolescents were recruited from three secondary schools located in the French-speaking region of Belgium, in the cities of Louvain-la-Neuve (Ecole Martin V), Bastogne (Institut Notre-Dame Séminaire) and Lessines (Athénée Royal René Magritte). Students from Louvain-la-Neuve had access to an indoor nonchlorinated swimming pool sanitised by the copper-silver method, while students of the two other schools visited only indoor chlorinated swimming pools. Of the 1,200 adolescents who were contacted in these three schools, a total of $1,137(94.8 \%)$ returned the questionnaire. Among them, 857 had the written agreement of their parents to participate in the study, giving an overall participation rate of $71.4 \%$. There was little variation in participation rate between the three schools (Louvain-la-Neuve 72.0\%; Bastogne 70.6\%; and Lessines $72.1 \%$ ), and little variation in the participation rate between females $(70.8 \%)$ and males $(72.2 \%)$. The present study nevertheless included more females than males, largely because of the school population at Bastogne that was comprised of $66 \%$ females. In total, eight adolescents who did not give blood were excluded, along with two others because of incomplete information provided in the questionnaire. Comparison of questionnaires from the participants $(n=847)$ and the nonparticipants $(n=280)$ did not reveal any significant differences in the prevalence of doctor-diagnosed asthma, nor in the proportions of adolescents having a backyard pool or having attended an outdoor pool during their holidays. The study protocol was approved by the Ethics Committee of the Faculty of Medicine of the Catholic University of Louvain (Louvain-la-Neuve) and complied with all applicable requirements of the international regulations.

\section{Questionnaire}

Parents were asked to complete a questionnaire inquiring about the family history of allergic diseases, the health of their child and the child's exposure to a variety of environmental or lifestyle factors likely to affect the studied outcomes. The questionnaire also contained questions about the attendance of indoor or outdoor swimming pools with the school, as a recreational or sport activity, and at home or during holidays. For each type of swimming pool, parents were asked to specify whether the pool was disinfected using chlorine or another disinfectant and to provide, for each year that an estimate of the number of hours per week and, when appropriate (e.g. during holidays), the number of weeks per year that their child had attended the pool. Returned questionnaires were checked and completed by interviewing the adolescents during their examination in schools.

\section{Examination of adolescents}

Adolescents were examined in schools between March and May 2006. After the measurement of height and body weight and the collection of one blood sample on a dry tube $(10 \mathrm{~mL})$, the concentration of nitric oxide (NO) was measured in exhaled air with the NIOX ${ }^{\mathrm{TM}}$ analyser (Aerocrine AB, Solna, Sweden) by following the guidelines of the American Thoracic Society [19]. Exercise-induced bronchoconstriction (EIB) was screened by measuring the fall in forced expiratory volume in one second (FEV1) after $6 \mathrm{~min}$ of indoor running with submaximal effort [20].

\section{Serum analyses}

The total and aeroallergen-specific IgE concentrations in serum were measured using the Immulite $\operatorname{IgE}$ kit (Diagnostic Products Company, Los Angeles, CA, USA). Specific IgE was screened against the following allergens: house dust mite (Dermatophagoides pteronyssinus); cat epithelium; dog dander; moulds (Penicilium notatum, Cladosporium herbarum, Aspergillus fumigata, Candida albicans and Alternaria tenui); tree pollen mixture (Alnus incana, Betula verrucosa, Corylus avellana, Quercus alba and Salix caprea); grass pollen mixture (Antoxanthum odoratum, Sacale cereale, Holcuns lanatus, Lolium perenne and Phleum pretense); and herbaceous pollen mixture (Chenopodium album, Solidago virgaurea, Urtica dioca, Artemisia absinthium and Artemisia vulgaris).

\section{Study outcomes}

Asthma was defined as either "ever asthma", corresponding to asthma diagnosed by a physician at any time in life, or as "current asthma", corresponding to physician-diagnosed asthma that was under medication or associated with a positive EIB test at the time of the study. The EIB test was considered positive when the exercise caused a fall of FEV1 by $\geqslant 10 \%$, which is the standard criterion for diagnosing exercise-induced asthma in athletes [21]. The exhaled NO test was considered 
positive when the concentration of $\mathrm{NO}$ in exhaled air was $>30 \mathrm{ppb}$. Sensitisation against the specific aeroallergens was defined as a serum concentration of specific $\operatorname{IgE}>0.35 \mathrm{kIU} \cdot \mathrm{L}^{-1}$.

\section{Statistical analysis}

Continuous variables are presented as median (interquartile range). The Mann-Whitney test was used for two-group comparisons and the Kruskal-Wallis nonparametric ANOVA test for comparing more than two groups. Categorical variables were compared by the Chi-squared test or by a Chi-squared test for trend for assessing the significance of exposureresponse relationships. Backward logistic regression models were implemented in order to analyse associations between outcomes and swimming pool attendance. Backward selection started with a model including all potential control variables and executing each step by deleting the least significant predictor until the model only contained variables with $p<0.20$. This level of significance was used as inclusion criterion to ensure that all important confounders end up in the model [22]. The following control variables were tested: age; sex; body mass index (BMI); ethnicity (white/nonwhite); birth weight; maternal smoking during pregnancy; breastfed; day nursery attendance; maternal and/or paternal history of asthma or allergy; total $\operatorname{IgE}$ in serum $\left(100 \mathrm{kIU} \cdot \mathrm{L}^{-1}\right)$; number of older siblings; socioeconomic status based on mother's and father's educational level; house cleaning with bleach; parental smoking at home; active smoking; regular practice of a sport other than swimming; and cumulative swimming pool attendance over lifetime or before the age of $7 \mathrm{yrs}$. The attendance at swimming pool before the age of 7 yrs was tested by adjusting for the cumulative attendance after 7 yrs. For each type of pool, the lifetime cumulative attendance was categorised as low, average or high, according to whether it was $0-100 \mathrm{~h}, 100-500 \mathrm{~h}$ or $>500 \mathrm{~h}$, respectively. These cut-off points were divided by two to create the corresponding categories for the pool attendance before the age of 7 yrs. The crude and adjusted odds ratios (ORs) for these categories were calculated using the occurrence of the outcome in adolescents having never attended the studied outdoor swimming pool as the reference level. Independent variables were checked for the absence of multicollinearity by calculating the tolerance and variance inflation factors for each variable. In order to test interactions between cumulative pool attendance and atopy, these logistic regression analyses were repeated by stratifying adolescents according to their total or aeroallergen-specific IgE in serum. Cut-off points for total serum $\operatorname{IgE}$ concentrations were 25,50 and $100 \mathrm{kIU} \cdot \mathrm{L}^{-1}$; these were derived from the median serum $\operatorname{IgE}$ concentration in the whole population $\left(50.1 \mathrm{kIU} \cdot \mathrm{L}^{-1}\right)$. Results were considered as statistically significant at $\mathrm{p}<0.05$ (two-sided).

\section{RESULTS}

Table 1 displays the characteristics of the adolescents who participated to the study. Students from the three schools were, on average, the same age (15 yrs). Sex ratio was close to one, except at Bastogne, where the school population included more females. Socioeconomic status, as evaluated on the basis of parental education, was higher at Louvain-la-Neuve than in the other schools and this was reflected in several lifestyle factors, such as BMI, exposure to tobacco smoke, breastfeeding and day care attendance. Because they had access to an indoor copper-silver sanitised pool, students of Louvain-la-Neuve had spent much less time in indoor chlorinated pools than their peers at Bastogne and Lessines, but their attendance of outdoor chlorinated pools was higher. There were, by contrast, no significant differences between the three schools regarding the prevalence of ever or current asthma, parental asthma, or in the rate of sensitisation to aeroallergens, with the exception of pollen. The rate of sensitisation to pollen, and similarly the mean values of total serum IgE and exhaled NO, were indeed slightly higher at Louvain-la-Neuve than in the two other schools. The lifetime cumulative attendance of a residential or nonresidential outdoor pool considered separately was not significantly different between adolescents with ever diagnosed asthma and those without asthma diagnosis $(p=0.61$ and 0.10 , respectively). The total lifetime attendance of outdoor pools, in contrast, was significantly greater among adolescents with ever asthma than in those who had no asthma diagnosis (median 348 versus 203 h; $\mathrm{p}=0.008$ ).

Table 2 shows that the cumulative attendance of a residential or nonresidential pool is associated with a rather similar pattern of asthma risks. Adolescents with the highest attendance $(>500 \mathrm{~h}$ ) of either type of pool showed approximately a two- to three-fold increase in the risk of ever or current asthma, even though the odds for current asthma did not reach statistical significance. In contrast, the risk of elevated exhaled NO was significantly increased only among adolescents with the highest attendance of a residential pool. Other significant predictors identified in these analyses were: total serum $\mathrm{IgE}$ (ever or current asthma and exhaled NO); parental asthma (ever or current asthma); house cleaning with bleach (ever or current asthma); sex (exhaled NO); and maternal smoking during pregnancy (ever asthma). It is noteworthy that none of the other variables that differed between the three schools (i.e. sex, BMI, parental education, active or passive smoking, breastfed and day care attendance) entered in the models, even at $\mathrm{p}<0.20$. Interestingly, house cleaning with bleach was found to exert a protective effect against the risk of ever asthma (OR 0.48, 95\% confidence interval (CI) 0.27-0.87; $\mathrm{p}=0.016$ ) and current asthma (OR 0.33, 95\% CI 0.14-0.76; $p=0.01$ ). All these associations persisted with pool attendance indices cumulated from birth to the age of 7 yrs (data not shown).

Since the attendance of a residential or a nonresidential pool similarly increased asthma risk, the attendance at both types of pools were combined in order to increase the numbers of subjects in the different pool attendance categories. This allowed for the assessment of exposure-response relationships while stratifying adolescents according to their family history of asthma and their attendance of indoor chlorinated pools. Figure 1 shows that the prevalence of ever and current asthma increased in a dose-dependent manner with increasing lifetime outdoor pool attendance, both when considering all adolescents (fig. 1a and c) or only those without parental asthma (fig. $1 \mathrm{~b}$ and $\mathrm{d}$ ). The sequential exclusion of adolescents with increasing attendance of indoor pools noticeably strengthened these relationships, especially for ever asthma. For instance, among adolescents having attended an indoor pool for $<250 \mathrm{~h}$, the prevalence of ever and current asthma were 4 and 9 times higher, respectively, in those with the highest outdoor pool attendance $(>500 \mathrm{~h})$ compared with their peers who had never swum in an outdoor pool (both $\mathrm{p}<0.001$; fig. 1a). Quite 
TABLE 1 Characteristics of adolescents

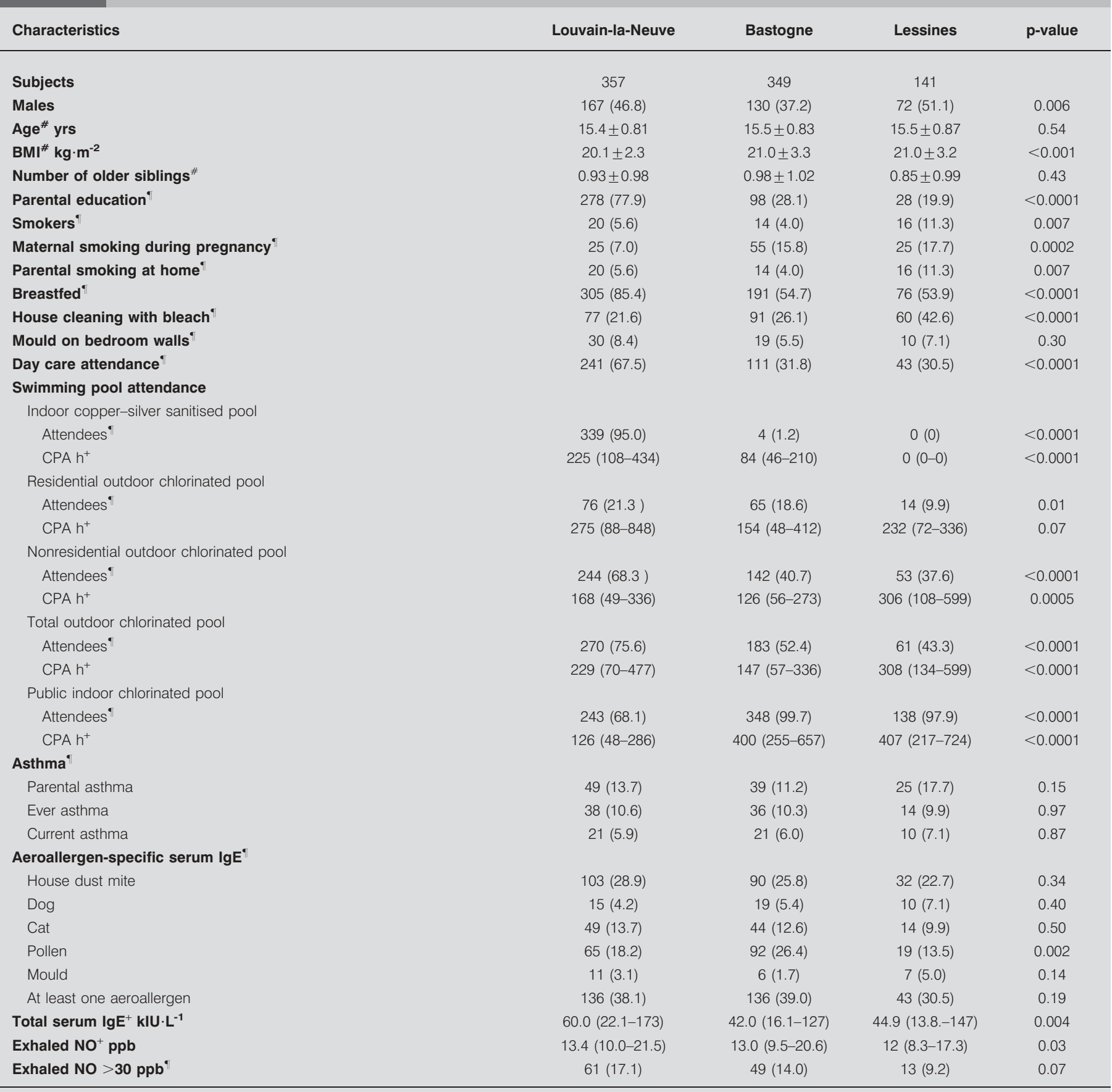

Data are expressed as $n, n(\%)$, mean \pm SD or median (interquartile range). BMI: body mass index; CPA: lifetime cumulative pool attendance; lg: immunoglobulin. ${ }^{\#}$ : twosided unpaired t-test; ${ }^{\bullet}$ : two-sided Chi squared test; ${ }^{+}$: two-sided Mann-Whitney U-test.

remarkably, among adolescents without parental asthma, the prevalence of current asthma increased almost linearly with the outdoor pool attendance by a factor of $>10$ (fig. 1b). Also, the group of adolescents with the lowest exposure to pool chlorine, i.e. those who never swam in outdoor chlorinated pools and had attended an indoor chlorinated pool for $<100 \mathrm{~h}$, had a prevalence of current asthma that was four times lower than in the rest of the population $((1.6 \%) 2$ out of 125 versus $(7.3 \%) 53$ out of $722 ; p=0.02)$.

The influence of atopic status on asthma risks associated with outdoor pool attendance was studied by calculating the odds for ever and current asthma in adolescents stratified according to atopy defined on the basis of total IgE or aeroallergen-specific 


\begin{tabular}{|c|c|c|c|c|c|c|c|c|c|c|}
\hline \multirow{3}{*}{\multicolumn{2}{|c|}{$\begin{array}{l}\text { TABLE } 2 \\
\text { Indicator }\end{array}$}} & \multirow{3}{*}{$\begin{array}{l}\text { Outdoor pool } \\
\text { attendance } \mathrm{h}\end{array}$} & \multirow{2}{*}{\multicolumn{4}{|c|}{ Residential outdoor chlorinated pool }} & \multirow{2}{*}{\multicolumn{4}{|c|}{ Nonresidential outdoor chlorinated pool }} \\
\hline & & & & & & & & & & \\
\hline & & & $\begin{array}{c}\text { Crude OR } \\
(95 \% \mathrm{Cl})\end{array}$ & $p$-value & $\begin{array}{l}\text { Adjusted OR } \\
\qquad(95 \% \mathrm{Cl})\end{array}$ & p-value & $\begin{array}{l}\text { Crude OR } \\
(95 \% \mathrm{Cl})\end{array}$ & p-value & $\begin{array}{l}\text { Adjusted OR } \\
\qquad(95 \% \mathrm{Cl})\end{array}$ & p-value \\
\hline \multirow{3}{*}{\multicolumn{2}{|c|}{ Ever asthma\# }} & $>0-100$ & $0.52(0.16-1.72)$ & 0.29 & $0.43(0.12-1.51)$ & 0.19 & $0.68(0.34-1.36)$ & 0.28 & $0.60(0.29-1.23)$ & 0.16 \\
\hline & & $>100-500$ & $1.11(0.49-2.53)$ & 0.80 & $1.30(0.56-3.07)$ & 0.54 & $1.29(0.76-2.20)$ & 0.35 & $1.17(0.67-2.05)$ & 0.59 \\
\hline & & $>500$ & $2.37(1.05-5.37)$ & 0.04 & $2.44(1.01-5.90)$ & 0.05 & $2.28(1.14-4.54)$ & 0.02 & $2.09(0.99-4.41)$ & 0.05 \\
\hline \multirow{3}{*}{\multicolumn{2}{|c|}{ Current asthma }} & 0 & $1.0(1.0-1.0)$ & & $1.0(1.0-1.0)$ & & $1.0(1.0-1.0)$ & & $1.0(1.0-1.0)$ & \\
\hline & & $>0-100$ & $0.28(0.04-2.06)$ & 0.21 & $0.24(0.03-1.86)$ & 0.17 & $0.97(0.44-2.15)$ & 0.94 & $0.81(0.35-1.92)$ & 0.64 \\
\hline & & $>100-500$ & $1.00(0.35-2.88)$ & 1.00 & $1.26(0.42-3.73)$ & 0.68 & $1.40(0.72-2.70)$ & 0.32 & $1.19(0.59-2.42)$ & 0.62 \\
\hline \multicolumn{2}{|c|}{ Exhaled $\mathbf{N O}>30 \mathrm{ppb}^{+}$} & $>500$ & $2.87(1.40-5.87)$ & 0.004 & $2.88(1.35-6.12)$ & 0.006 & $1.19(0.59-2.40)$ & 0.99 & $0.99(0.47-2.09)$ & 0.98 \\
\hline \multicolumn{11}{|c|}{$\begin{array}{l}\text { \#: odds ratios (ORs) for ever asthma were adjusted for total serum immunoglobulin (Ig)E, parental asthma, sex, maternal smoking during pregnancy, house cleaning with } \\
\text { bleach, the presence of mould on bedroom walls, the number of older siblings and the attendance at the other type of outdoor pool. ?: ORs for current asthma were } \\
\text { adjusted for total serum IgE, parental asthma, sex, maternal smoking during pregnancy, house cleaning with bleach and the attendance at the other type of outdoor pool. } \\
+: \text { ORs for exhaled nitric oxide were adjusted for total serum IgE, parental allergy, sex and house cleaning with bleach. The numbers of subjects among the referents and } \\
\text { the three pool attendance categories were as follows. Residential pools: } 692,54,63 \text { and 38; nonresidential pools: 408, 164, } 208 \text { and } 67 \text {. }\end{array}$} \\
\hline
\end{tabular}

serum IgE. This analysis was performed by excluding adolescents with parental asthma $(n=113)$ and those with an indoor pool attendance $>500 \mathrm{~h}(\mathrm{n}=214)$ in order to specifically assess the effects of outdoor pool attendance. When atopy was defined on the basis of total serum IgE, outdoor chlorinated pool attendance was associated with an increased risk of ever or current asthma only in children with total serum $\operatorname{IgE}$ $>25 \mathrm{kIU} \cdot \mathrm{L}^{-1}$. Above this threshold, outdoor pool attendance and serum IgE level strongly interacted to cause a dosedependent increase in asthma risk. From the ORs given in table 3, it can be estimated that there is an overall $100-200 \%$ increase of ever or current asthma risk with each 100-h increase in outdoor pool attendance, depending on the level of serum IgE and of pool attendance. When atopy was defined on the basis of allergen-specific serum IgE, risks of ever and current asthma significantly increased with outdoor pool attendance only among sensitised subjects, but the ORs were approximately two times lower than those observed in subjects with high concentrations of serum IgE (data not shown).

It was ascertained that the interactions between outdoor pool attendance and total serum $\operatorname{IgE}$ were not specific for one school, in particular that of Louvain-la-Neuve, whose students had mainly attended the copper-silver sanitised pool. As shown in table 4, the interactions between outdoor pool attendance and total serum IgE persisted, and even appeared stronger, when students of Louvain-la-Neuve and those of Bastogne and Lessines were analysed separately. The interaction was particularly remarkable at Louvain-la-Neuve, probably because the cumulative exposure of referents to indoor chlorinated pools was much lower than in the two other schools (median 24 versus $256 \mathrm{~h}$ ).
There were no significant associations between the risks of sensitisation to aeroallergens and the attendance of a residential or nonresidential outdoor pool when cumulatively assessed over a lifetime. However, when studying associations with pool attendance during early childhood, it was found that adolescents who had regularly attended a residential pool before the age of $7 \mathrm{yrs}$ were more likely to be sensitised to aeroallergens, and particularly to cat or house dust mite allergens (table 5). Risks of asthma and of elevated exhaled NO were particularly increased among these adolescents. In contrast, attendance at a nonresidential pool during early childhood was not associated with an increased risk of sensitisation to aeroallergens.

When considering the whole population, no significant association emerged between asthma and the attendance at indoor chlorinated pools, whether cumulative over lifetime or during early childhood. However, when considering adolescents with a low exposure to outdoor pools $(<100 \mathrm{~h})$, the highest indoor pool attendance $(>500 \mathrm{~h})$ was associated with a significant increase in the risk of ever asthma (OR 5.7, 95\% CI 1.2-26.7; $\mathrm{p}=0.02$ ) and a nonsignificant increase in the risk of current asthma (OR 2.17, 95\% CI 0.84-5.61; $\mathrm{p}=0.11$ ). The attendance of indoor chlorinated pools did not influence the risks of respiratory allergies. The current authors also found no significant associations between any of the studied outcomes and the attendance of the copper-silver sanitised pool.

\section{DISCUSSION}

The present study shows that regular attendance at an outdoor chlorinated pool, at home or during holidays, is associated with an exposure-dependent increase in the risk of asthma. 

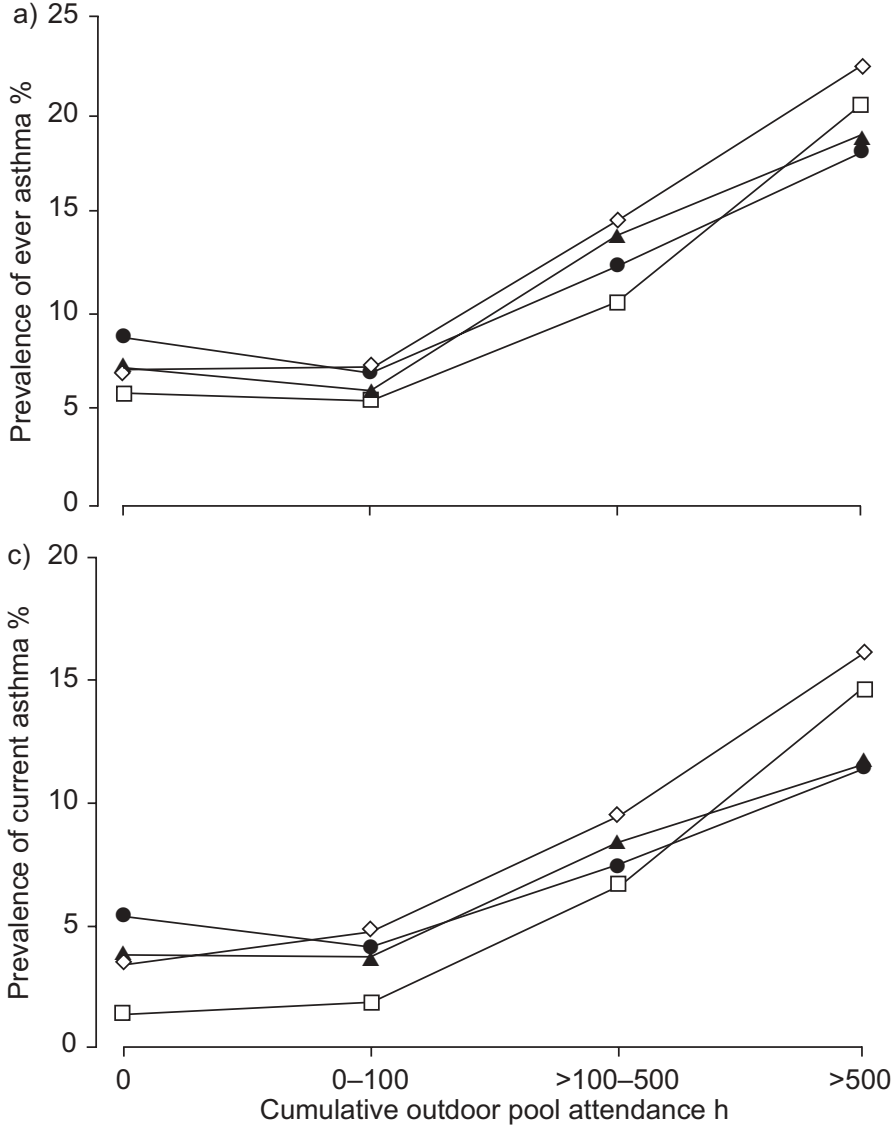

b)

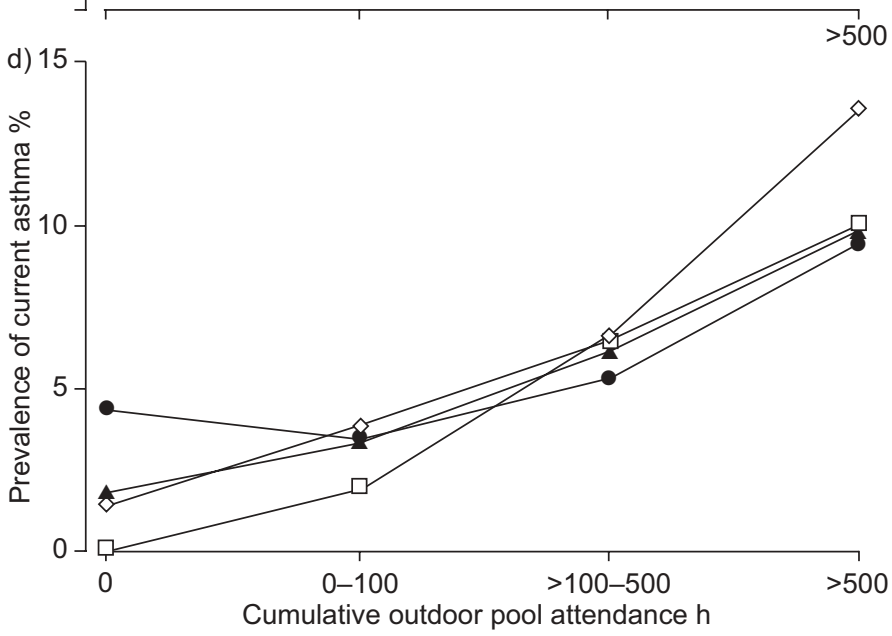

FIGURE 1. Prevalence of ever asthma and current asthma in all adolescents (a and c) and in those without parental asthma (b and d) according to their lifetime attendance of outdoor chlorinated swimming pools, considering either all subjects $(\bullet)$ or subjects with cumulative indoor pool attendance lower than $100(\square), 250(\diamond)$ and $500 \mathrm{~h}(\mathbf{\Lambda})$. The numbers of subjects in these four categories were respectively 847, 235, 410 and 633 in a) and c) and 734, 211, 357 and 547 in b) and d). p-values correspond to the Chi-squared test for trend. a) $\bullet, \diamond$ and $\mathbf{\Lambda}: p<0.001 ; \square: p=0.01$. b) $\bullet: p=0.01 ; \square: p=0.015 ; \diamond$ and $\mathbf{\Lambda}: p<0.001 . c) \bullet: p=0.01 ; \square: p=0.001 ; \diamond: p<0.001 ; \boldsymbol{\Lambda}$ $p=0.005$. d) $\bullet: p=0.03 ; \square: p=0.015 ; \diamond: p<0.001 ; \mathbf{\Lambda}: p=0.005$.

Adolescents having regularly attended a residential pool were also more likely to have a positive in the exhaled nitric oxide test result and, when attendance was during infancy, to be sensitised against cat or house dust mite allergens. These associations cannot be explained by differences in socioeconomic level and related lifestyle factors, such as BMI, whether breastfed or exposure to tobacco smoke. They are also unlikely to result from a reverse causation due to a greater propensity of adolescents to attend an outdoor pool because they had been diagnosed with asthma. The cumulative attendance at either type of outdoor pools was indeed not significantly different between adolescents diagnosed with asthma and their peers without asthma. Furthermore, as there were no public outdoor pools in the studied centres, the hypothesis of a reverse causation would imply that parents would have been encouraged to install a backyard pool or to spend holidays in places having an outdoor pool by the fact that their child had asthma. Such a confounding appears especially improbable as asthmatics are advised to swim not in outdoor pools but in indoor pools, in which the warm and humid atmosphere is less likely to trigger asthma symptoms [23].

As expected, total serum $\operatorname{IgE}$ and parental asthma ranked as the strongest predictors of ever and current asthma. Maternal smoking during pregnancy emerged as a significant predictor only for ever asthma while exposure to parental smoking at home had no influence. This is consistent with earlier studies showing that the associations between passive smoking and asthma risks are the strongest during early childhood and then disappear with increasing age [24]. Interestingly, the present study confirms that house cleaning with bleach protects against the risk of asthma, probably by decreasing the exposure to indoor allergens and harmful microbial agents, such as fungal products or endotoxins $[25,26]$. This protection afforded by bleach is not inconsistent with the increased asthma risks associated with swimming pools, since children living in a house cleaned with bleach are not directly in contact with chlorination products as they are when playing or swimming in an chlorinated pool [25].

The present study reveals new insights into the pool factors responsible for respiratory problems in swimmers. It is now possible to exclude the contention that asthma and allergy risks associated with swimming pool attendance are caused by swimming itself, since none of the studied outcomes showed a significant association with the attendance of the copper-silver sanitised pool. This conclusion is supported by the fact that some outcomes, such as exhaled nitric oxide or sensitisation to 


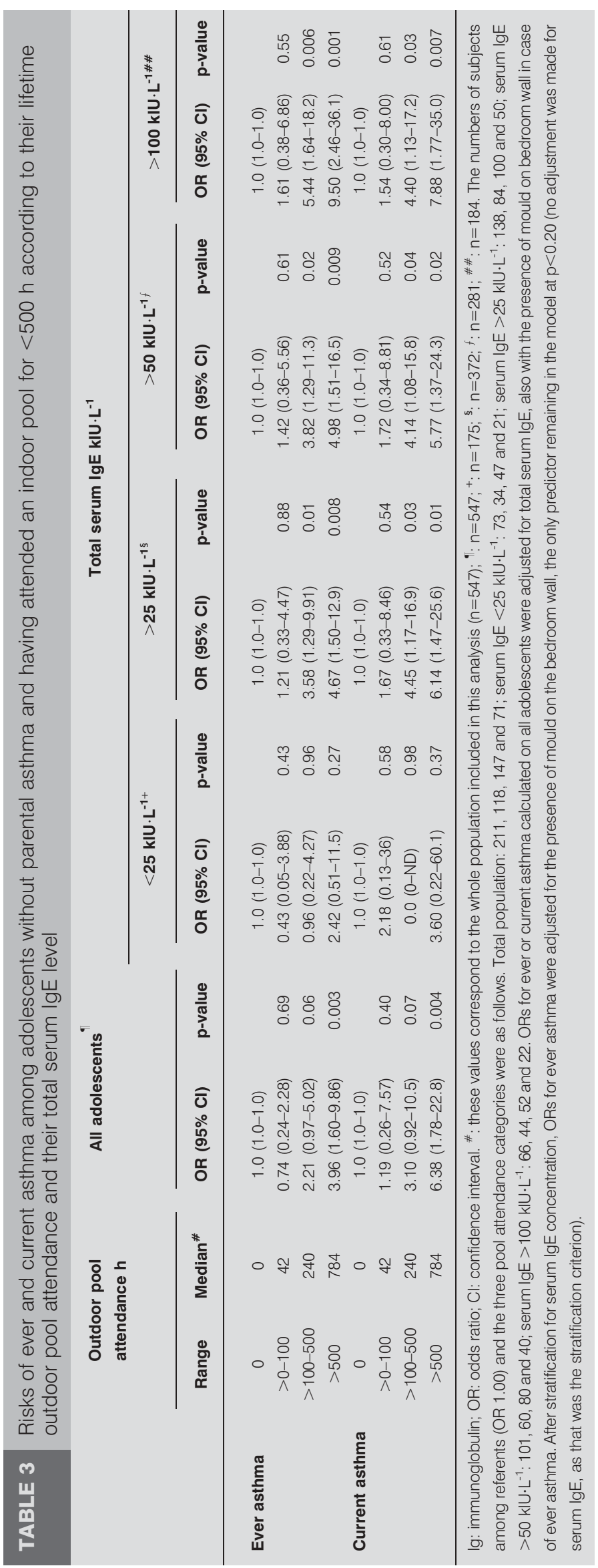

house dust mites, were already significantly associated with the outdoor pool attendance accumulated before the age of 7 yrs, i.e. when most adolescents could not really swim. The present authors believe that the cause of respiratory effects found in the current study has to be sought among the chlorination products that are present in pool water or that build up at the surface of the pool. Trichloramine is unlikely to be responsible for these effects as this highly volatile gas is very quickly dispersed into the atmosphere, explaining why open-air swimming pools do not have the characteristic "chlorine" smell of indoor pools. The most concentrated and reactive chlorine compound to which swimmers are exposed in outdoor pools is hypochlorous acid, i.e. the active chlorine itself. Hypochlorous acid is a well-known lung toxicant [27] and, at concentrations used in pool water (1-3 ppm) [1, 2], this powerful oxidising agent could quite conceivably affect the airways of swimmers when they inhale aerosols or small volumes of water. Pool water and the air just above the water surface also contains a variety of reactive chlorination byproducts including chloramines, trihaloacetic acid or trihaloacetonitriles [28]. Although they are usually less concentrated in pool water than active chlorine, there is no doubt that these chemicals also contribute to the burden of oxidants or irritants inhaled by swimmers.

In addition to the increased risk of asthma, the present study has identified associations between the attendance of a residential outdoor pool and the risks of respiratory allergies or airways inflammation measured by the exhaled nitric oxide test. The fact that such associations were not observed with nonresidential outdoor pools is interesting. This difference is indeed in accordance with the mechanism by which chlorinebased oxidants could promote allergic sensitisation and that consists of a disruption in epithelial barriers, facilitating the delivery of antigens $[3,10]$. To be induced, such a mechanism implies a certain coincidence between the exposure to allergens and the exposure to chlorination products, a coincidence that for indoor allergens is more likely to occur when the pool is at home than in a resort or in a summer house. This is particularly obvious for pets like cats that usually do not follow their owner on holidays. Exposure to house dust mite allergen is probably also lower in places of holidays, owing to the drier climate or to the more efficient of destruction of allergens by professional laundering [29].

Although chlorine and hypochlorous acid are among the most powerful oxidants to be found, the possibility that these chemicals could adversely affect organs of swimmers in contact with pool air or water has so far received little attention. However, these chlorine-based oxidants are known to cause oxidative damage to the epithelial and endothelial layers in contact with chlorinated water or aerosols [30-33]. While for any other air pollutant such effects would be considered as unacceptable, most regulatory bodies regard them as simply a source of discomfort for swimmers [2]. This lack of concern for the oxidant effects of these chemicals on swimmers is reflected by the current guidelines that allow concentrations of active chlorine up to $3 \mathrm{ppm}$ and even higher (e.g. after a shock treatment). The current authors believe that such high concentrations of active chlorine are not necessary and may even be hazardous to the swimmers, particularly the youngest, who can spend 


\begin{tabular}{|c|c|c|c|c|c|c|c|c|c|c|}
\hline & Range & Median $^{\#}$ & \multicolumn{2}{|c|}{$<25 \mathrm{kIU}^{-\mathrm{L}^{-1}}$} & \multicolumn{2}{|c|}{$>25 \mathrm{kIU} \cdot \mathrm{L}^{-1}$} & \multicolumn{2}{|c|}{$>50 \mathrm{kIU} \cdot \mathrm{L}^{-1}$} & \multicolumn{2}{|c|}{$>100 \mathrm{kIU} \cdot \mathrm{L}^{-1}$} \\
\hline \multirow[t]{3}{*}{ Louvain-la-Neuve } & 0 & 0 & $1.0(1.0-1.0)$ & & $1.0(1.0-1.0)$ & & $1.0(1.0-1.0)$ & & $1.0(1.0-1.0)$ & \\
\hline & $>0-100$ & 35 & $1.77(0.14-22.5)$ & 0.86 & $0.69(0.03-14.3)$ & 0.81 & $0.74(0.04-14.8)$ & 0.85 & $0.85(0.05-15.5)$ & 0.91 \\
\hline & $>100-500$ & 280 & $0.80(0.06-10.5)$ & 0.84 & $11.7(1.11-124)$ & 0.04 & $10.9(1.07-110)$ & 0.54 & $10.9(1.13-106)$ & 0.04 \\
\hline Lessines $^{+}$ & $>500$ & 725 & 0.0 (0-ND) & 1.0 & $3.9(0.82-18.6)$ & 0.09 & $4.76(0.87-26.0)$ & 0.07 & $12.8(1.69-97.8)$ & 0.01 \\
\hline
\end{tabular}

Ig: immunoglobulin; OR: odds ratio; Cl: confidence interval;. ": These values correspond to the adolescents without parental asthma and with a cumulated indoor chlorinated pool attendance $<500 \mathrm{~h} .{ }^{\circ}: \mathrm{n}=285 ;^{+}: \mathrm{n}=262$. The numbers of subjects among referents (OR 1.00$)$ and the three pool attendance categories were as follows Louvain-la-Neuve: total population: $78,72,90$ and 45 ; serum IgE $<25 \mathrm{kIU} \cdot \mathrm{L}^{-1}: 25,15,33$ and 11; serum IgE $>25 \mathrm{kIU} \cdot \mathrm{L}^{-1}: 53,57,57$ and 34 ; serum IgE $>50 \mathrm{kIU} \cdot \mathrm{L}^{-1}: 40$, 42, 46 and 27; serum IgE $>100 \mathrm{kIU} \cdot \mathrm{L}^{-1}: 27,29,29$ and 16. Bastogne-Lessines: total population: 133, 46, 57 and 26; serum IgE $<25 \mathrm{kIU} \cdot \mathrm{L}^{-1}: 48,19,14$ and 10; serum IgE $>25 \mathrm{kIU} \cdot \mathrm{L}^{-1}: 85,27,43$ and 16 ; serum $\lg \mathrm{E}>50 \mathrm{kIU} \cdot \mathrm{L}^{-1}: 61,18,34$ and 13 ; serum IgE $>100 \mathrm{kIU} \cdot \mathrm{L}^{-1}: 39,15,23$ and 6 . ORs were adjusted for the presence of mould on bedroom walls, the only predictor entering in the model at $p<0.20$ (no adjustment was made for serum IgE, as that was the stratification criterion).

TABLE 5 Risks of asthma, increased exhaled nitric oxide and of sensitisation to aeroallergens associated with the attendance at a residential outdoor chlorinated swimming pool before the age of 7 yrs during a cumulative time of $>50 \mathrm{~h}$

\begin{tabular}{|c|c|c|c|c|c|c|}
\hline & No & Yes & Unadjusted & $p$-value & Adjusted & p-value \\
\hline Subjects n & 804 & 43 & & & & \\
\hline Cat lgE & $97(12.1)$ & $10(23.3)$ & $2.20(1.05-4.61)$ & 0.04 & $2.57(1.21-5.47)$ & 0.014 \\
\hline Dog $\lg E$ & $42(5.2)$ & $2(4.7)$ & $0.89(0.21-3.78)$ & 0.87 & $1.13(0.26-4.92)$ & 0.87 \\
\hline Mould IgE & $1(2.3)$ & $23(2.9)$ & $0.81(0.11-6.1)$ & 0.84 & $1.09(0.14-8.46)$ & 0.93 \\
\hline Pollen IgE & $169(21.0)$ & $7(16.3)$ & $0.73(0.32-1.67)$ & 0.46 & $0.82(0.35-1.89)$ & 0.63 \\
\hline Ever asthma & $77(9.6)$ & $11(25.6)$ & $3.25(1.57-6.70)$ & 0.001 & $3.49(1.61-7.57)$ & 0.002 \\
\hline \multicolumn{7}{|c|}{ 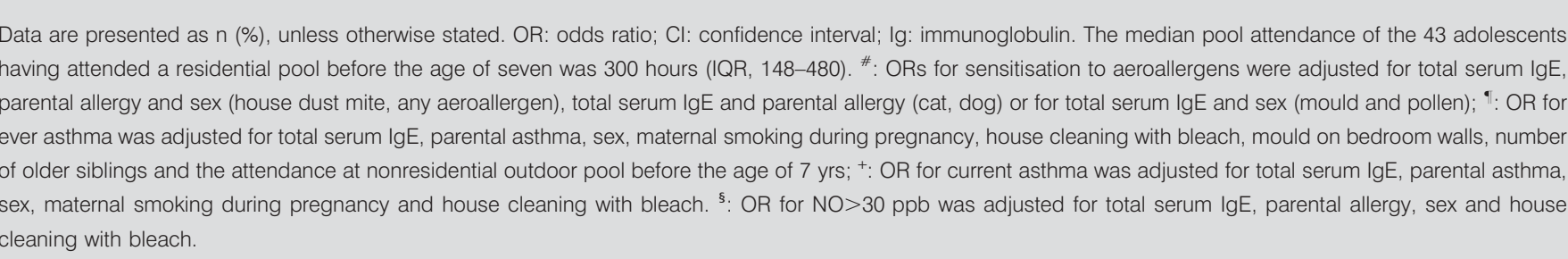 } \\
\hline
\end{tabular}


hours playing in outdoor pools. The experience with public indoor swimming pools teaches us that in a well-designed and operated pool, an adequate disinfection can be achieved with active chlorine concentrations in the range 0.5-1.0 ppm and even with lower concentrations if one refers to the German standards (0.3-0.7 ppm) [16]. There are no reasons to think that concentrations of active chlorine in this range should not also be sufficient to disinfect outdoor pools, especially the residential pools where the infectious risks, primarily due to faecal contamination, are normally lower than in public pools.

The principal strength of the present study lies in the use of robust outcome measures, which allowed considerable reduction in the risk of recall or response bias. Although the present study required a blood sampling in schools, a relatively good response rate $(71.4 \%)$ could be achieved, which further reduced the risks of selection bias. The present authors have also taken advantage of the existence in Belgium of an indoor copper-silver sanitised pool that was in activity for $>20$ yrs. Since in industrialised countries swimming pool attendance has become a very popular, and even a compulsory, activity in schools, this was indeed a rather unique opportunity to recruit a control group with no or a minimal exposure to chlorination products while being well matched with the exposed group according to swimming practice.

The present authors assessment of exposure to pool chlorine was, however, more limited, as no choice existed but to use the information provided by the questionnaire filled by the parents. However, it is unlikely that the responses of the parents to the questions about outdoor attendance could have been biased by the health of their child or the perception they had of the benefits or risks of swimming in chlorinated pools. First, the parents were blinded to the tested hypothesis since outdoor pool attendance was only one of the many environmental or lifestyle factors that were addressed by the questionnaire. Secondly, the hypothesis that outdoor pool attendance could cause adverse effects is probably very far from the belief that parents had when they offered their child the possibility to swim in an outdoor pool, at home or during the holidays. Thirdly, even assuming a bias in the parental responses to the questionnaire, it is difficult to imagine that this bias could have distorted the analysis in proportion to the serum IgE level of adolescents, generating consistent relationships between asthma prevalence and cumulative pool attendance across the categories of increasing serum IgE.

In summary, the present study shows that the attendance at outdoor chlorinated swimming pools, at home or during holidays, is associated with an exposure-dependent increase in the risk of asthma, especially among children with higher serum immunoglobulin E levels. Attendance at a residential outdoor pool also appears to increase the risk of airways inflammation and sensitisation to some indoor aeroallergens. Since these associations were not found with the attendance of the copper-silver pool, they are most likely due to some airways damage caused by chlorine-based oxidants added to pool water or released at the surface of the pool as aerosols or gases. These findings may have important implications in countries where outdoor pools are very common.

\section{REFERENCES}

1 Center for Disease Control. Healthy Swimming. www.cdc.gov/healthyswimming/ph_chlorine.htm Date last modified: May 1, 2008. Date last accessed: July 23, 2008.

2 Guidelines for Safe Recreational Waters. Volume 2. Swimming pools and similar recreational-water environments. Geneva, World Health Organization, 2006.

3 Bernard A. Chlorination products: emerging links with allergic diseases. Curr Med Chem 2007; 14: 1771-1782.

4 Haag JR, Giese RG. Effects of swimming pool water on the cornea. JAMA 1983; 249: 2507-2508.

5 Momas I, Brette F, Spinasse A, Squinazi F, Dab W, Festy B. Health effects of attending a public swimming pool: follow up of a cohort of pupils in Paris. J Epidemiol Community Health 1993; 47: 464-468.

6 Pardo A, Nevo K, Vigiser D, Lazarov A. The effect of physical and chemical properties of swimming pool water and its close environment on the development of contact dermatitis in hydrotherapists. Am J Ind Med 2007; 50: 122-126.

7 Helenius IJ, Tikkanen HO, Sarna S, Haahtela T. Asthma and increased bronchial responsiveness in elite athletes: atopy and sport event as risk factors. J Allergy Clin Immunol 1998; 101: 646-652.

8 Helenius I, Rytilä P, Sarna S, et al. Effect of continuing or finishing high-level sports on airway inflammation, bronchial hyperresponsiveness, and asthma: a 5-year prospective follow-up study of 42 highly trained swimmers. J Allergy Clin Immunol 2002; 109: 962-968.

9 Carbonnelle S, Francaux M, Doyle I, et al. Changes of serum pneumoproteins caused by short-term exposures to nitrogen trichloride in indoor chlorinated pools. Biomarkers 2002; 7: 464-478.

10 Bernard A, Carbonnelle S, Michel O, et al. Lung hyperpermeability and asthma prevalence in schoolchildren: unexpected associations with the attendance of indoor chlorinated pools. Occup Environ Med 2003; 60: 385-394.

11 Bernard A, Carbonnelle S, De Burbure C, Michel O, Nickmilder M. Chlorinated pool attendance, atopy and the risk of asthma during childhood. Environ Health Perspect 2006; 114: 1567-1573.

12 Bernard A, Carbonnelle S, Dumont X, Nickmilder M. Infant swimming, pulmonary epithelium integrity and the risk of allergic and respiratory diseases later in childhood. Pediatrics 2007; 119: 1095-1103.

13 Nickmilder M, Bernard A. Ecological association between childhood asthma and availability of indoor chlorinated swimming pools in Europe. Occup Environ Med 2007; 64: 37-46.

14 Lagerkvist B, Bernard A, Blomberg A, et al. Pulmonary epithelial integrity in children - relationship to ambient ozone exposure and swimming pool attendance. Environ Health Perspect 2004; 112: 1767-1772.

15 Stav D, Stav M. Asthma and whirlpool baths. N Engl J Med 2005; 353: 1635-1636.

16 Kohlhammer $\mathrm{Y}$, Doring A, Schafer T, Wichmann HE, Heinrich J, for the KORA study group, Swimming pool attendance and hay fever rates later in life. Allergy 2006; 61: 1305-1309.

17 Thickett KM, McCoach JS, Gerber JM, Sadhra S, Burge PS. Occupational asthma caused by chloramines in indoor swimming pool air. Eur Respir J 2002; 19: 827-832. 
18 Jacobs JH, Spaan S, van Rooy GB, et al. Exposure to trichloramine and respiratory symptoms in indoor swimming pool workers. Eur Respir J 2007; 29: 690-698.

19 American Thoracic Society. Recommendations for standardized procedures for the online and offline measurement of exhaled lower respiratory nitric oxide and nasal nitric oxide in adults and children. Am J Respir Crit Care Med 1999; 160: 2104-2117.

20 McFadden ER, Gilbert IA. Exercise-induced asthma. N Engl J Med 1994; 330: 1362-1367.

21 Dickinson JW, Whyte GP, McConnell AK, Nevill AM, Harries MG. Mid-expiratory flow versus FEV1 measurements in the diagnosis of exercise induced asthma in elite athletes. Thorax 2006; 61: 111-121.

22 Greenland S. Modelling and variable selection in epidemiologic analysis. Am J Public Health 1999; 79: 340-349.

23 Nemery B, Hoet PHM, Nowak D. Indoor swimming pools, water chlorination and respiratory health. Eur Respir J 2002; 19: 790-793.

24 Gilmour I, Jaakola M, London S, Nel A, Rogers C. How exposure to environmental tobacco smoke, outdoor air pollutants, and increased pollen burdens influences the incidence of asthma. Environ Health Perspect 2006; 114: 627-633.

25 Nickmilder M, Carbonnelle S, Bernard A. House cleaning with chlorine bleach and the risks of allergic and respiratory diseases in children. Pediatr Allergy Immunol 2007; 18: 27-35.
26 Zock JP, Plana E, Jarvis D, et al. Hygiene and allergy: the use of bleach at home and atopic sensitisation in adults. Eur Respir J 2007; 30: Suppl. 51, E1423.

27 Hammerschmidt S, Büchler N, Wahn H. Tissue lipid peroxidation and reduced glutathione depletion in hypochlorite-induced lung injury. Chest 2002; 121: 573-581.

28 Erdinger L, Kirsch F, Sonnntag HG. [Irritating effects of disinfection by-products in swimming pools]. Zentralbl Hyg Umweltmed 1998; 200: 491-503.

29 Arlian LG, Vyszenski-Moher DL, Morgan MS. Mite and mite allergen removal during machine-washing of laundry. J Allergy Clin Immunol 2003; 111: 1269-1273.

30 Schraufstätter I, Browne K, Harris A, Hyslop P, Jackson J. Mechanisms of hypochlorite injury to target cells. J Clin Invest 1990; 85: 554-562.

31 Tatsumi T, Fliss H. Hypochlorous acid and chloramines increase endothelial permeability: possible involvement of cellular zinc. Am J Physiol 1994; 267: H1597-H1607.

32 Maldonado MJ. Corneal epithelial alterations resulting from use of chlorine-disinfected contact tonometer after myopic photorefractive keratectomy. Ophthalmology 1998; 105: 1546-1549.

33 Maurer JK, Molai A, Parker RD, et al. Pathology of ocular irritation with bleaching agents in the rabbit low-volume eye test. Toxicol Pathol 2001; 29: 308-319. 